\title{
The Effect of Hemoglobin Levels on Mortality in Pediatric Patients with Severe Traumatic Brain Injury
}

\author{
Kevin F. Yee, ${ }^{1}$ Andrew M. Walker, ${ }^{1}$ and Elaine Gilfoyle ${ }^{2}$ \\ ${ }^{1}$ Department of Anesthesia, Foothills Medical Centre, University of Calgary, 140329 Street NW, Calgary, AB, Canada T2N 2 T9 \\ ${ }^{2}$ Section of Critical Care, Department of Pediatrics, Faculty of Medicine, University of Calgary, Alberta Children's Hospital, \\ 2888 Shaganappi Trail NW, Calgary, AB, Canada T3B 6A8
}

Correspondence should be addressed to Kevin F. Yee; kyee99@gmail.com

Received 4 October 2015; Revised 11 April 2016; Accepted 30 May 2016

Academic Editor: Dario Olivieri

Copyright (C) 2016 Kevin F. Yee et al. This is an open access article distributed under the Creative Commons Attribution License, which permits unrestricted use, distribution, and reproduction in any medium, provided the original work is properly cited.

\begin{abstract}
Objective. There is increasing evidence of adverse outcomes associated with blood transfusions for adult traumatic brain injury patients. However, current evidence suggests that pediatric traumatic brain injury patients may respond to blood transfusions differently on a vascular level. This study examined the influence of blood transfusions and anemia on the outcome of pediatric traumatic brain injury patients. Design. A retrospective cohort analysis of severe pediatric traumatic brain injury (TBI) patients was undertaken to investigate the association between blood transfusions and anemia on patient outcomes. Measurements and Main Results. One hundred and twenty patients with severe traumatic brain injury were identified and included in the analysis. The median Glasgow Coma Scale (GCS) was 6 and the mean hemoglobin (Hgb) on admission was $115.8 \mathrm{~g} / \mathrm{L}$. Forty-three percent of patients $(43 \%)$ received at least one blood transfusion and the mean hemoglobin before transfusion was $80.1 \mathrm{~g} / \mathrm{L}$. Multivariable regression analysis revealed that anemia and the administration of packed red blood cells were not associated with adverse outcomes. Factors that were significantly associated with mortality were presence of abusive head trauma, increasing PRISM score, and low GCS after admission. Conclusion. In this single centre retrospective cohort study, there was no association found between anemia, blood transfusions, and hospital mortality in a pediatric traumatic brain injury patient population.
\end{abstract}

\section{Background}

Traumatic brain injury (TBI) is a leading cause of pediatric morbidity and mortality, accounting for approximately 60,000 hospitalizations and 7,400 deaths per year [1]. Substantial work has been done on anemia and transfusions in both adult and pediatric critically ill patients $[2,3]$. Several adult studies have shown an association between anemia, blood transfusions, and poorer outcomes in patients with severe TBI $[4,5]$.

While the current adult literature is gaining increasing evidence that there may be an adverse effect of transfusion on TBI patients, these results may not be generalizable to pediatric patients. Significant differences exist between pediatric and adult cerebral blood flow (CBF) in both normal and traumatic-injured states [6]. CBF and cerebral metabolic rates are higher in children [6]. In addition, pediatric patients have increased $\mathrm{CO}_{2}$ vasoreactivity and have a CBF autoregulation system that is easier to disrupt [6]. The type of blood being used in pediatric patients may also affect the outcome. Lacroix et al. determined that pediatric patients often received leukocyte reduced blood compared to adults who did not [3].

Further, pediatric TBI patients may respond to blood transfusions differently compared to adults. Figaji et al. reported that $79 \%$ of their pediatric TBI patients demonstrated an improvement in brain oxygenation after a transfusion [7]. This is in contrast to the $57 \%$ of adult TBI patients that had an improvement in brain oxygenation after a blood transfusion [8].

The Transfusion Requirements in Pediatric Intensive Care Unit (TRIPICU) trial demonstrated that adopting a restrictive blood transfusion threshold of a hemoglobin (Hgb) level of $70 \mathrm{~g} / \mathrm{L}$ in stable critically ill children results in no difference in mortality, but they did not look at TBI patients specifically [3]. Comprehensive evidence-based guidelines have been created to optimize the management of 
pediatric TBI patients, but there is no consensus on when to transfuse pediatric TBI patients [9]. We performed a retrospective cohort study of pediatric severe TBI patients admitted to the pediatric intensive care unit (PICU) to determine if there is any association between anemia and blood transfusions with mortality in these patients.

\section{Materials and Methods}

Approval from the Conjoint Health Research Ethics Board at the University of Calgary was obtained (Study ID REB130095) and informed consent was waived. Patients with a diagnosis of TBI admitted to the Alberta Children's Hospital (ACH) PICU between January 2001 and December 2012 had their charts analyzed by the study investigators. Inclusion criteria were $<18$ years of age, admission to $\mathrm{ACH}$ PICU with diagnosis of TBI or skull fracture, and initial Glasgow Coma Scale (GCS) of $\leq 8$. Exclusion criteria included patient obeying commands within 12 hours of admission, patient death within 12 hours of admission (with injuries likely so severe that blood transfusions would be unlikely to alter their course), nontraumatic etiology to explain decreased level of consciousness (e.g., alcohol or drugs), and concomitant traumatic quadriparesis (unable to assess GCS). These criteria were modelled after a similar study in adult TBI patients [5]. No sampling was done and all patients who met eligibility criteria were included for analysis.

Data was collected on standardized case report forms. Aside from standard demographic information, additional data collected included patient initial hemoglobin and mean hemoglobin for up to the next 7 days after admission; type and severity of the injury characterized by initial vital signs and GCS scores; mechanism of injury; surgical procedures performed; and the presence of signs indicative of raised intracranial pressure (ICP) on CT scans, as reported by a radiologist. At our institution, all patients with severe TBI receive radiologic studies as part of their workup, but not all patients receive invasive ICP monitoring. Presence of intracranial hemorrhage and other injuries was also collected. Recording of patient management data included transfusions in the first 14 days, use of mannitol or hypertonic saline, therapeutic hypothermia, and administration of neuromuscular blockade. Length of hospital and ICU stay along with allcause mortality within 30 days was used as our primary endpoints.

A database containing all previously admitted pediatric patients diagnosed from the desired time period was created. All patients with diagnosis codes of brain injury, traumatic cerebral edema, skull fractures, wounds to head, face, or scalp, and intracranial bleeding admitted to the PICU for more than 24 hours were included in the initial screening.

Presented patient demographic and clinical data are expressed as the mean \pm SD or median with interquartile range (IQR) dependent on data normality as assessed using the Shapiro-Wilk test $(p<0.05)$. Given the potential practice change of the publication of the landmark transfusion threshold study in critically ill children by Lacroix et al., we stratified the patients according to whether they were admitted prior to or after 2007 [3]. Significant differences

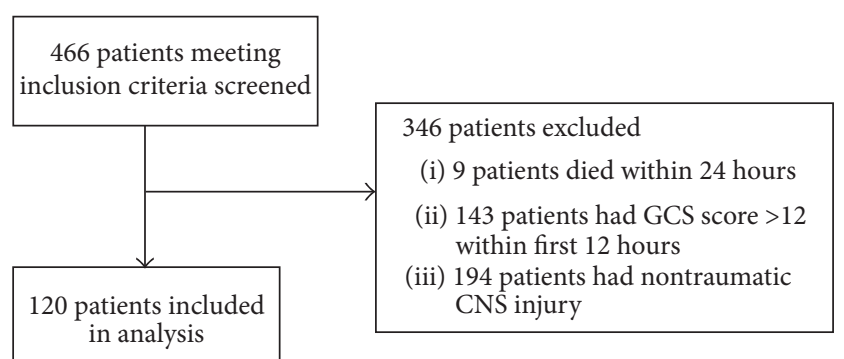

FIgURE 1: Total number of patients screened and included into the study. TBI: traumatic brain injury. GCS: Glasgow Coma Scale. CNS: central nervous system.

$(p<0.05)$ in continuous demographic and clinical variables between those transfused and not transfused were assessed using the independent samples $t$-test or Mann Whitney $U$ test upon failure of data normality. Significant differences $(p<0.05)$ in categorical variables were assessed using the chisquare test for association or Fisher's exact test.

An exploratory model to predict the probability of mortality was developed using logistic regression. Seven independent variables (age, presence of suspected nonaccidental trauma (NAT), PRISM III score, RBC transnfusion, admission GCS score, admission Hgb, and 7-day mean Hgb) were initially considered as possible covariates. Prior to model construction, a correlation of $\rho=0.65$ was found between admission Hgb and 7-day mean Hgb using Spearman's rank coefficient. Univariate logistic regression was used to assess the strength of admission and 7-day mean Hgb in predicting the probability of mortality. Nagelkerke $R^{2}$ values were 0.1 and 0.02 for admission and 7-day mean $\mathrm{Hgb}$, respectively, supporting the consideration of admission $\mathrm{Hgb}$ as a covariate in our predictive logistic regression model. Additionally, an interaction term of RBC infusion * admission Hgb was also considered as a possible covariate.

The criteria of variable selection for model inclusion followed that of Hosmer Jr. et al. [10]. Briefly, variable selection began with univariate analysis of each independent variable via chi-square analysis for categorical variables (presence of NAT and RBC infusion) and univariate logistic regression for continuous variables. Candidate variables were considered if $p<0.2$ and as such, all seven covariates were included in an initial predictive model. Results from the initial predictive model of mortality guided the generation of a refined model using only covariates that presented with $p<0.05$ [10]. The refined, reduced covariate model was compared to the full covariate model using the likelihood ratio chi-square test. All statistical tests were completed using IBM SPSS 20.0 statistics software (IBM, Armonk, NY, USA).

\section{Results}

A total of 466 patients with TBI were screened for possible study inclusion (Figure 1). One hundred and twenty met the inclusion criteria. Fifty-three patients (44\%) received blood transfusions. Of these patients, the average number of transfusions was 1.67 . 
TABLE 1: Demographic information. GCS: Glasgow Coma Scale. Hgb: hemoglobin. PRISM III: pediatric risk of mortality III score. SD: standard deviation. Values represented as " $x(y)$ " are the median (interquartile range). Values with " $x \pm y$ " are mean \pm SD.

\begin{tabular}{|c|c|c|c|}
\hline Clinical variable & Patients transfused $(n=53)$ & Patients not transfused $(n=67)$ & $p$ value \\
\hline Mean age (years) & $3.5(3.5)$ & $11.3(10.6)$ & $<\mathbf{0 . 0 0 5}$ \\
\hline Male gender (\%) & $32(60)$ & $47(70)$ & 0.262 \\
\hline \multicolumn{4}{|l|}{ Year of admission (\%) } \\
\hline 2001-2006 & $28(52)$ & $34(51)$ & 0.821 \\
\hline $2007-2012$ & $25(48)$ & $33(49)$ & 0.821 \\
\hline \multicolumn{4}{|l|}{ Mechanism of injury } \\
\hline Motor vehicle accident & $23(43)$ & $26(39)$ & 0.611 \\
\hline Fall & $3(6)$ & $9(13)$ & 0.159 \\
\hline Assault & $10(19)$ & $10(15)$ & 0.565 \\
\hline Accidental & $17(32)$ & $22(33)$ & 0.930 \\
\hline Suspected nonaccidental trauma (\%) & $12(23)$ & $14(21)$ & 0.818 \\
\hline Mortality (\%) & $13(25)$ & $7(10)$ & 0.040 \\
\hline PRISM III score & $10.0(9)$ & $5.0(2)$ & $<0.005$ \\
\hline Mean ICU length of stay (days) & $6.0(6)$ & $3.0(2)$ & $<0.005$ \\
\hline Mean hospital length of stay (days) & $15.0(22)$ & $9.0(13)$ & 0.049 \\
\hline Patients with ICP monitor (\%) & $26(49)$ & $8(12)$ & $<0.005$ \\
\hline \multicolumn{4}{|l|}{ Mean daily ICP (mmHg) } \\
\hline Day 1 & $12.0(8)$ & $17.0(14)$ & 0.624 \\
\hline Day 2 & $14.0(8)$ & $16.0(12)$ & 0.815 \\
\hline Day 3 & $16.0(8)$ & $18.0(8)$ & 0.477 \\
\hline Day 4 & $14.0(8)$ & $15.0(13)$ & 0.673 \\
\hline Day 5 & $14.0(10)$ & $18.0(53)$ & 0.622 \\
\hline Day 6 & $18.0 \pm 10.8$ & & \\
\hline Day 7 & $16.5 \pm 4.4$ & & \\
\hline Admission Glasgow Coma Scale (GCS) & $3.0(4)$ & $6.0(4)$ & 0.009 \\
\hline \multicolumn{4}{|l|}{ Surgical procedures performed (\%) } \\
\hline 0 & $27(51)$ & $55(82)$ & $<0.005$ \\
\hline 1 & $10(19)$ & $7(10)$ & 0.189 \\
\hline 2 & $16(30)$ & $5(7)$ & 0.001 \\
\hline Presence of raised ICP on imaging (\%) & $32(60)$ & $20(30)$ & 0.001 \\
\hline Presence of intracranial hemorrhage (\%) & $39(74)$ & $39(58)$ & 0.079 \\
\hline \multicolumn{4}{|c|}{ Intracranial hypertension medical management (\%) } \\
\hline Mannitol admin & $21(40)$ & $10(15)$ & 0.002 \\
\hline $3 \% \mathrm{NaCl}$ admin & $6(11)$ & $4(6)$ & 0.292 \\
\hline Hypothermia & $8(15)$ & $9(13)$ & 0.795 \\
\hline Use of neuromuscular blockade (\%) & $21(40)$ & $19(28)$ & 0.194 \\
\hline Admission $\mathrm{Hgb}(\mathrm{g} / \mathrm{L})$ & $101.6 \pm 24.6$ & $126.7 \pm 13.9$ & $<0.005$ \\
\hline Nadir Hgb in first 7 days $(\mathrm{g} / \mathrm{L})$ & $83.0(20)$ & $105.0(18)$ & $<0.005$ \\
\hline Mean Hgb over first 7 days (g/L) & $106.8(12)$ & $115.8(14.5)$ & $<0.005$ \\
\hline Mean number of transfusions & $1.67 \pm 1.78$ & & \\
\hline
\end{tabular}

Patient demographic and clinical data is summarized in Table 1. Statistically significant differences $(p<0.05)$ between patients transfused and not transfused were noted in age, mortality, PRISM III score, ICU length of stay, hospital length of stay, patients with ICP monitor, initial GCS, surgical procedures performed, presence of raised ICP on imaging, mannitol administration, admission Hgb, nadir $\mathrm{Hgb}$ in first 7 days, and mean $\mathrm{Hgb}$ over first 7 days. Of the patients who received blood transfusions, 26 patients received invasive ICP monitoring, while 8 patients in the nontransfused group did. One patient was excluded from the length-of-stay calculations as they were transferred to another facility before they were discharged, but their data was used in the main analysis.

The reduced covariate logistic regression model (Table 2) to predict the probability of mortality was statistically significant $\left(\chi^{2}=47.4 ; p<0.05\right)$ and included presence of 
TABLE 2: Statistical outputs of initial exploratory logistic regression model with seven covariates (a) and final reduced covariate (b) logistic regression model to predict the probability of mortality.

(a)

\begin{tabular}{|c|c|c|c|c|c|}
\hline Variables & $\beta$ & $\begin{array}{c}\text { Standard } \\
\text { error }\end{array}$ & $p$ value & Odds ratio & $\begin{array}{c}95 \% \text { CI odds } \\
\text { ratio (lower- } \\
\text { upper) }\end{array}$ \\
\hline NAT suspected & 2.434 & 0.989 & 0.014 & 11.40 & $1.64-79.25$ \\
\hline PRISM III score & 0.222 & 0.072 & $<0.005$ & 1.25 & $1.08-1.44$ \\
\hline Age & 0.039 & 0.094 & 0.679 & 1.04 & $0.87-1.25$ \\
\hline Admission GCS & -0.303 & 0.226 & 0.179 & 0.74 & $0.47-1.15$ \\
\hline Initial Hgb & -0.057 & 0.042 & 0.174 & 0.95 & $0.87-1.03$ \\
\hline $\mathrm{RBC}$ transfusion & -5.688 & 5.366 & 0.289 & 0.00 & $0.00-125.17$ \\
\hline $\begin{array}{l}\text { Initial Hgb } * \mathrm{RBC} \\
\text { transfusion }\end{array}$ & 0.046 & 0.045 & 0.302 & 1.05 & $0.96-1.14$ \\
\hline Constant & 3.094 & 5.468 & 0.572 & 22.06 & \\
\hline
\end{tabular}

$R^{2}=0.586 ; \chi^{2}=51.1$.

(b)

\begin{tabular}{lccccc}
\hline Variables & $\beta$ & $\begin{array}{c}\text { Standard } \\
\text { error }\end{array}$ & $p$ value & Odds ratio & $\begin{array}{c}\text { 95\% CI odds ratio } \\
\text { (lower-upper) }\end{array}$ \\
\hline NAT suspected & 2.198 & 0.730 & $<0.005$ & 9.011 & $2.155-37.684$ \\
PRISM III score & 0.276 & 0.060 & $<0.005$ & 1.317 & $1.171-1.482$ \\
Constant & -5.294 & 0.937 & $<0.005$ & 0.005 & \\
\hline
\end{tabular}

$R^{2}=0.552 ; \chi^{2}=47.4$.

NAT: nonaccidental trauma; GCS: Glasgow Coma Scale; Hgb: hemoglobin; RBC: red blood cell.

suspected NAT and PRISM III score as significant $(p<0.05)$ covariates:

Log odds (mortality)

$$
\begin{aligned}
= & -5.294+(2.198 * \text { presence of suspected NAT }) \\
& +(0.276 * \text { PRISM III score })
\end{aligned}
$$

Odds ratios were 9.01 (95\% CI: 2.16-37.68) and 1.32 (95\% CI: 1.17-1.48) for presence of suspected NAT and PRISM III score, respectively. The model explained $55.2 \%$ of the variance in mortality (Nagelkerke $R^{2}$ ) and correctly classified $89.1 \%$ of patients. Sensitivity and specificity were $50 \%$ and $97 \%$, respectively. The likelihood ratio chi-square test was not significant $\left(\chi^{2}=3.70 ; p=0.594\right)$.

\section{Discussion}

In this retrospective cohort study of 120 pediatric patients with severe TBI admitted to our PICU, it was found that hemoglobin at time of admission, administration of blood transfusions, and 7-day average hemoglobin after admission were not associated with adverse outcomes. Significant variables that were associated with increased mortality were the presence of suspected NAT and increasing PRISM score.

It should be noted that there was a significant difference in the patients that were transfused and those that were not. The patients receiving blood transfusions tended to be younger and less well. Despite these differences, there was no clinical outcome difference associated with hemoglobin levels and transfusions.

The goals of TBI management are to prevent secondary insults to the brain after the initial injury, including injury caused by hypoxia and hypotension $[9,11]$. Hemoglobin is the major carrier of oxygen in the systemic circulation and there are many conflicting studies examining the relationship between anemia and TBI outcome. Sekhon and colleagues showed that a mean 7-day hemoglobin of less than $90 \mathrm{~g} / \mathrm{L}$ resulted in increased hospital mortality in severe adult TBI patients [5].

In a large retrospective study of adult TBI patients, Salim and colleagues demonstrated that anemia and correction of it by blood transfusion are associated with increased mortality [4]. Similarly, Warner and colleagues found that adult patients receiving transfusions for moderate anemia after suffering TBI had long-term reduced function at 6 months [12]. In our study, we were not able to record good neurologic outcome data since they were often not documented. Currently, no association between administering blood transfusions and positive outcomes can be made in adult TBI patients and our study supports this assertion in pediatric TBI patients. However, one cannot separate the effects of transfusion with the effects of anemia on outcome in TBI. This is a limitation of the retrospective design of our study, as well as the published adult studies.

Similar to Sekhon and colleagues, we chose to study measured exposure to hemoglobin levels by looking at the initial and average hemoglobin for up to 7 days after admission. A variety of methods have been used to observe hemoglobin 
levels, but 7 days is thought to capture the occurrence of peak ICP [13]. Like their study, we also captured transfusions as a time-insensitive dichotomous variable that cannot take into context the circumstances surrounding the administration of blood. We recognize that our results may be affected by a survival bias since we did not collect the exact time of death for those patients who died. It is well recognized that, in pediatric critical care, decisions to withdraw life support and the subsequent timing of this withdrawal are heavily affected by the patient's social and cultural backgrounds, so the time of death may not always be based on purely medical circumstances. This makes the link between time of death and severity of illness very difficult in our context $[14,15]$.

Other limitations of this study include the retrospective nature and the long period of time during which patient information was gathered. During this period of data collection, management strategies may have changed over time, which may have also affected our results. Having said that, our multivariate logistic regression analysis did not show an association between other management strategies and outcomes. All patients reviewed were admitted to a single centre suggesting that our results may not be entirely generalizable to other hospitals. However, the fact that our study found associations between NAT, GCS, and PRISM scores suggests that our study population is similar to other pediatric TBI studies that identified these as factors as well [16-18].

In conclusion, initial admission $\mathrm{Hgb}$ and mean $\mathrm{Hgb}$ values averaged over seven days after admission to PICU were not found to be strong predictors of mortality in children with severe TBI. No difference in outcome can be demonstrated when patients are transfused. Based on our study, we cannot advocate for deviation from the widely accepted transfusion threshold of $7 \mathrm{~g}$ per deciliter in critically ill pediatric patients presenting with TBI [3]. Prospective evaluation of the association among anemia, transfusion, and outcome should be undertaken to further define any possible relationship.

\section{Disclosure}

The study institution where this research was carried out is Section of Critical Care, Department of Pediatrics, Faculty of Medicine, University of Calgary, Alberta Children's Hospital, 2888 Shaganappi Trail NW, Calgary, AB, Canada T3B 6A8.

\section{Competing Interests}

The authors declare that they have no competing interests.

\section{Acknowledgments}

The authors would like to thank Dr. Jong Rho and the Section of Neurology in the Department of Pediatrics in the Cumming School of Medicine at the University of Calgary for providing departmental funds to support this study.

\section{References}

[1] R. M. Stanley, B. K. Bonsu, W. Zhao, P. F. Ehrlich, A. J. Rogers, and H. Xiang, "US estimates of hospitalized children with severe traumatic brain injury: implications for clinical trials," Pediatrics, vol. 129, no. 1, pp. e24-e30, 2012.

[2] P. C. Hébert, G. Wells, M. A. Blajchman et al., "A multicenter, randomized, controlled clinical trial of transfusion requirements in critical care," The New England Journal of Medicine, vol. 340, no. 6, pp. 409-417, 1999.

[3] J. Lacroix, P. C. Hébert, J. S. Hutchison et al., "Transfusion strategies for patients in pediatric intensive care units," The New England Journal of Medicine, vol. 356, no. 16, pp. 1609-1619, 2007.

[4] A. Salim, P. Hadjizacharia, J. DuBose et al., "Role of anemia in traumatic brain injury," Journal of the American College of Surgeons, vol. 207, no. 3, pp. 398-406, 2008.

[5] M. S. Sekhon, N. McLean, W. R. Henderson, D. R. Chittock, and D. E. G. Griesdale, "Association of hemoglobin concentration and mortality in critically ill patients with severe traumatic brain injury," Critical Care, vol. 16, no. 4, article R128, 2012.

[6] Y. Udomphorn, W. M. Armstead, and M. S. Vavilala, "Cerebral blood flow and autoregulation after pediatric traumatic brain injury," Pediatric Neurology, vol. 38, no. 4, pp. 225-234, 2008.

[7] A. A. Figaji, E. Zwane, M. Kogels et al., "The effect of blood transfusion on brain oxygenation in children with severe traumatic brain injury," Pediatric Critical Care Medicine, vol. 11, no. 3, pp. 325-331, 2010.

[8] D. A. Zygun, J. Nortje, P. J. Hutchinson, I. Timofeev, D. K. Menon, and A. K. Gupta, "The effect of red blood cell transfusion on cerebral oxygenation and metabolism after severe traumatic brain injury," Critical Care Medicine, vol. 37, no. 3, pp. 1074-1078, 2009.

[9] P. M. Kochanek, N. Carney, P. D. Adelson et al., "Guidelines for the acute medical management of severe traumatic brain injury in infants, children, and adolescents," in Introduction. Pediatric Critical Care Medicine, vol. 13, chapter 1, pp. S1-S2, 13, 2nd edition, 2012.

[10] D. W. Hosmer Jr., S. Lemeshow, and R. X. Sturdivant, Applied Logistic Regression, John Wiley \& Sons, Hoboken, NJ, USA, 2013.

[11] Brain Trauma Foundation, American Association of Neurological Surgeons, Congress of Neurological Surgeons et al., "Guidelines for the management of severe traumatic brain injury. I. Blood pressure and oxygenation," Journal of Neurotrauma, vol. 24, supplement 1, pp. S7-S13, 2007.

[12] M. A. Warner, T. O’Keeffe, P. Bhavsar et al., "Transfusions and long-term functional outcomes in traumatic brain injury," Journal of Neurosurgery, vol. 113, no. 3, pp. 539-546, 2010.

[13] N. Stocchetti, A. Colombo, F. Ortolano et al., "Time course of intracranial hypertension after traumatic brain injury," Journal of Neurotrauma, vol. 24, no. 8, pp. 1339-1346, 2007.

[14] D. Garros, R. J. Rosychuk, and P. N. Cox, "Circumstances surrounding end of life in a pediatric intensive care unit," Pediatrics, vol. 112, no. 5, article e371, 2003.

[15] K. L. Meert, L. Keele, W. Morrison et al., "End-of-life practices among tertiary care PICUs in the United States: a multicenter study," Pediatric Critical Care Medicine, vol. 16, no. 7, pp. e231e238, 2015.

[16] M. Bahloul, A. Chaari, I. Chabchoub et al., "Outcome analysis and outcome predictors of traumatic head injury in childhood: analysis of 454 observations," Journal of Emergencies, Trauma and Shock, vol. 4, no. 2, pp. 198-206, 2011. 
[17] F. Kipfmueller, H. Wyen, M. A. Borgman, P. C. Spinella, S. Wirth, and M. Maegele, "Epidemiology, risk stratification and outcome of severe pediatric trauma," Klinische Padiatrie, vol. 225, no. 1, pp. 34-40, 2013.

[18] K. J. Deans, P. C. Minneci, W. Lowell, and J. I. Groner, "Increased morbidity and mortality of traumatic brain injury in victims of nonaccidental trauma," Journal of Trauma and Acute Care Surgery, vol. 75, no. 1, pp. 157-160, 2013. 


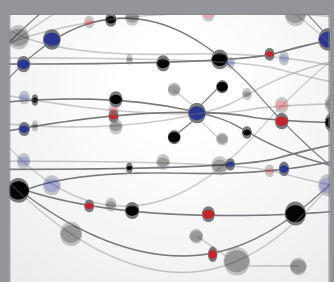

The Scientific World Journal
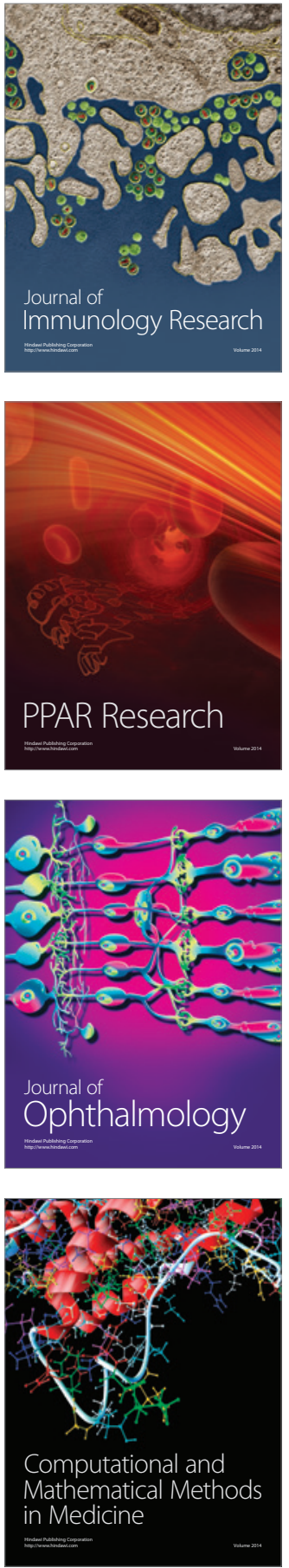

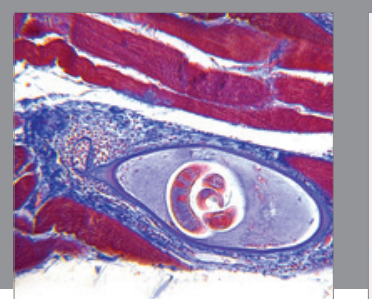

Gastroenterology Research and Practice

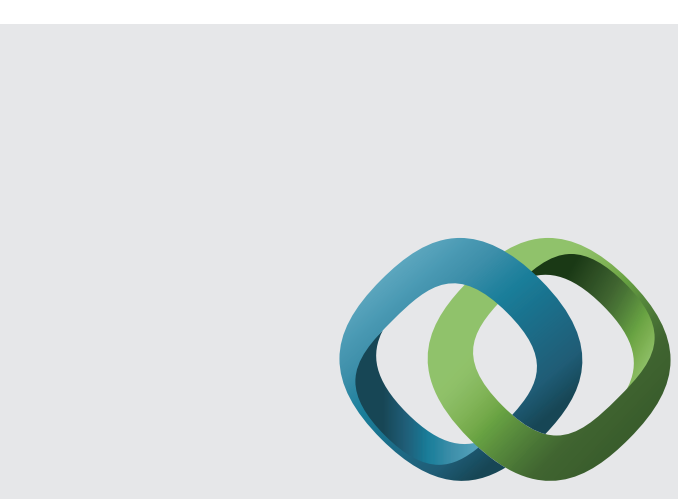

\section{Hindawi}

Submit your manuscripts at

http://www.hindawi.com
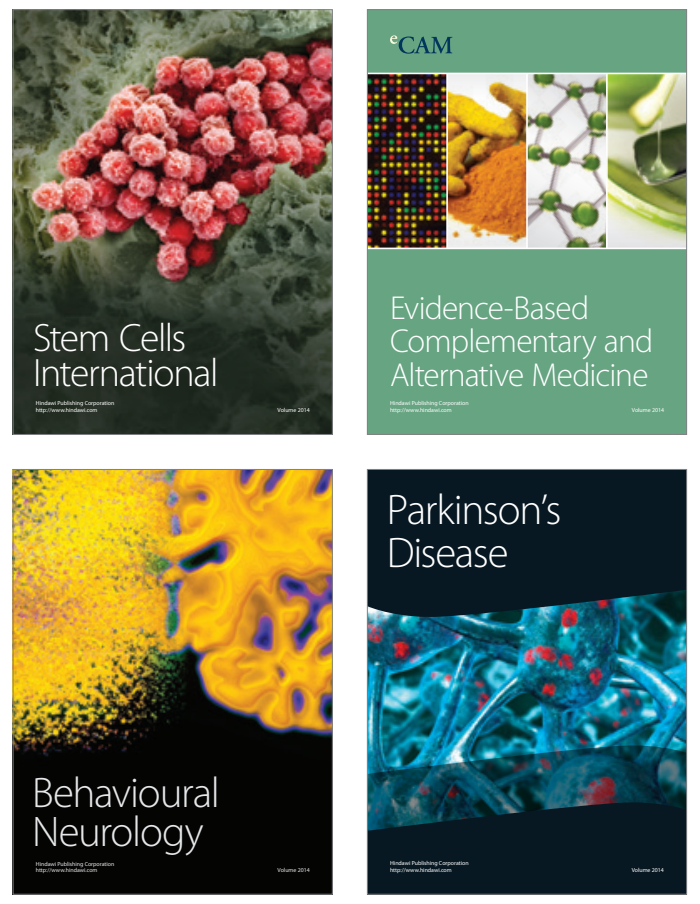
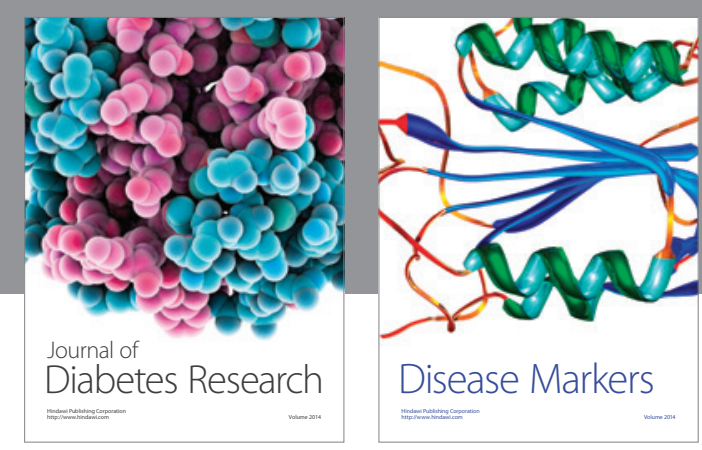

Disease Markers
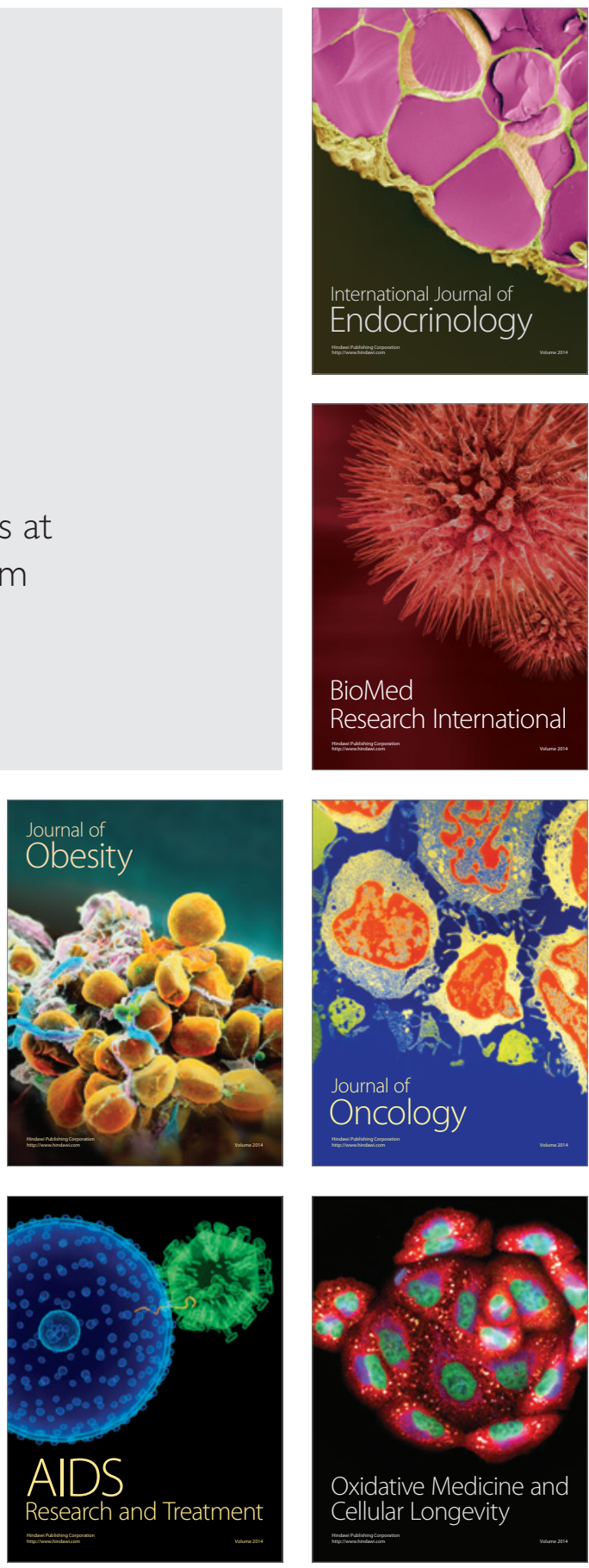